\title{
Relationship of Oral Bacterial Load Over One Year of Smoking Cessation
}

\author{
Sunghyun $\mathrm{Kim}^{1}$, Min-Seock Seo ${ }^{2}$, and Soo-Jeong Hwang ${ }^{3, \dagger}$ \\ 'Department of Clinical Laboratory Science, College of Health Sciences, Catholic University of Pusan, Busan 46252, \\ ${ }^{2}$ Department of Conservative Dentistry, Wonkwang University Daejeon Dental Hospital, Daejeon 35233, \\ ${ }^{3}$ Department of Dental Hygiene, College of Medical Science, Konyang University, Daejeon 35365, Korea
}

\begin{abstract}
Background: Smoking exerts an adverse effect on the periodontal tissue by reorganizing the ecosystem of oral microorganisms and is considered to be an important factor in the development of periodontal disease. Although cross-sectional studies on smokers and non-smokers have been attempted to investigate the microbial differences in periodontal oral cavity, only few studies have been conducted to investigate the changes in oral microorganisms during smoking cessation. The purpose of this study was to investigate the changes of bacteria in saliva and gingival crevicular fluid (GCF) over a period of one year among 11 smokers trying to quit smoking.

Methods: Eleven smokers trying to quit smoking visited the clinic at baseline, two weeks, two months, four months, six months, and 12 months to give saliva and GCF samples. The amounts of 16S rRNA, Porphyromonas gingivalis, Treponema denticola, Prevotella intermedia, Fusobacterium nucleatum subsp. nucleatum, Streptococcus mutans, and Streptococcus sobrinus in saliva and GCF were quantified using real-time polymerase chain reaction TaqMan probe assay. The results were analyzed by nonparametric statistical analysis using Friedman test and Spearman correlation coefficient.

Results: After cessation of smoking, the amounts of $16 \mathrm{~S}$ rRNA corresponding to $P$. gingivalis, F. nucleatum, $P$. intermedia, and $T$. denticola in saliva decreased and then again increased significantly. The amount of $F$. nucleatum $16 \mathrm{~S}$ rRNA in GCF decreased significantly after smoking cessation. Positive correlations were observed between 16S rRNA and $F$. nucleatum and between $F$. nucleatum and $T$. denticola in saliva and GCF.

Conclusion: Even if the number of subjects in this study was small, we suggest that smoking cessation may reduce the total bacterial amount and $F$. nucleatum in GCF. However, the results regarding changes in the microbial ecosystem due to smoking or smoking cessation were inconsistent. Therefore, further in-depth studies need to be carried out.
\end{abstract}

Key Words: Bacteria, Periodontal disease, Smoking, Smoking cessation

\section{Introduction}

Smoking has an adverse effect on the periodontal tissue and is considered to be an important factor in the progression of periodontal disease. Previous studies have demonstrated the clinical impact of smoking, reporting that scaling and root planing were less effective in smokers than in non-smokers ${ }^{1)}$, and that periodontal treatment combined with smoking cessation resulted in greater reduction in average periodontal pocket depth ${ }^{2)}$.
When the amounts of matrix metalloproteinase 9 in gingival crevicular fluid (GCF) were measured in the smoking, non-smoking, and smoking cessation groups, significant differences between the groups were found after adjusting for age and gingival index ${ }^{3)}$, with the smoking group showing higher concentration of enzymes that destroy periodontal tissues.

In addition, other studies have reported that smoking reorganizes the ecosystem of oral microorganisms. Smoking was reported to stimulate formation of diverse 
and relatively unstable biofilms at gingival margins and subgingival spaces), while it was also reported that smokers are more susceptible to Porphyromonas gingivalis infection. In addition, smoking alters the expression of surface components of $P$. gingivalis to ultimately cause damage to immunoglobulins ${ }^{5}$. Shah et al. ${ }^{6}$ reported that smoking increases pro-inflammatory and oxidative stress response to virulence-enhanced commensal biofilms.

Assessing oral microorganisms or the health status of periodontal tissues in smokers and non-smokers through a cross-sectional study is less difficult than a longitudinal study. However, the follow-up survey study with smoking cessation as an intervening factor showed a low 12-month success rate in smoking cessation $(2.5 \sim 16.9 \%)^{7)}$, and thus, it was not easy to measure any changes. Although there was a study that regularly monitored patients undergone periodontal treatment to investigate the effects of periodontal treatment and smoking cessation ${ }^{8}$, there are limited studies that exclusively investigated changes in periodontal tissues during smoking cessation. The purpose of the present study was to observe and analyze changes in oral bacterial load during the smoking cessation process, without involvement of periodontal treatment, in 11 subjects who successfully quit smoking. These subjects were followed up for 12 months in public health center smoking cessation clinics.

\section{Materials and Methods}

\section{Study subjects and collection of GCF and saliva}

The study was performed on a population of 122 men who had signed a written consent to participate in the study and enrolled in public health center smoking cessation clinics. The exclusion criteria of the present study consisted of the following: those with uncontrolled systemic disease; those taking steroids or anti-inflammatory drugs during the study period or within three months prior to participating in the study; those using an oral rinse product during the study period or within three months prior to participating in the study; those receiving dental treatment during the study period; those with $\leq 20$ teeth, excluding the third molars; and those with any area of periodontal pocket $\geq 5.5 \mathrm{~mm}$. The subjects were instructed to visit the public health center at baseline, two weeks, and two, four, six, and 12 months. Due to a very high drop-out rate, only 26 subjects visited the public health center smoking cessation clinics at all time points. Among them, 15 subjects were unable to quit smoking and only 11 subjects successfully quit smoking for 12 months ${ }^{9}$. The success of smoking cessation was determined by verbal reports and using a carbon monoxide meter.

Each time a subject visited the health center, 2 to $4 \mathrm{ml}$ of unstimulated saliva sample was collected, while GCF was collected after removing biofilm and drying. Paper points were used 25 times for 1 minute absorption by intracrevicular "superficial" method from five interdental spots in maxillary anterior teeth and five interdental spots in mandibular anterior teeth. The samples were frozen immediately after collection at the health center and kept at $-20^{\circ} \mathrm{C}$ until the experiment was performed.

\section{Measurement of some oral bacterial load real-time polymerase chain reaction TaqMan probe assay}

Amounts of certain bacteria in saliva and GCF from mandibular anterior region were analyzed using real-time polymerase chain reaction (qPCR) TaqMan probe assay. Bacteria were received from the Korean Collection for Type Cultures (KCTC) and the following were selected: $P$. gingivalis (KCTC 5352, ATCC 33277), Treponema denticola (KCTC 15104, ATCC 35405), Prevotella intermedia (KCTC 5692, KCOM 1107), Fusobacterium nucleatum subsp. nucleatum (КСТC 2640, АСТC25586), Streptococcus mutans (KCTC 3065, ACTC 25175), and Streptococcus sobrinus (KCTC 3308, ACTC 27607). Molecular biological quantitative bacterial load test was performed at the Department of Clinical Laboratory Science, Catholic University of Pusan, with the primers and TaqMan probe sets prepared as suggested in the previous studies $^{10-12)}$.

\section{Statistics}

The cycle threshold $(\mathrm{Ct})$ values of real-time PCR were used in the analysis without converting them to bacterial count. This was based on the assumption that analysis 
using $\mathrm{Ct}$ values would be appropriate since specific bacteria may not be detected in some samples, and thus, the final variable was presented as $\mathrm{Ct}$ value in the table. Moreover, since the number of subjects was low $(n=11)$ and did not show normal distribution, parametric statistics could have been problematic, and thus, non-parametric statistics were used. For statistical analysis of changes in bacteria at each time point, the Friedman test was used, while the Spearman's correlation analysis was used for analyzing the correlation between variables. The statistical program IBM SPSS 20.0 (IBM Corp., Armonk, NY, USA) was used and the significance level was set to 0.05 .

\section{Results}

As shown in Table 1, with respect to the bacteria in saliva, the mean $\mathrm{Ct}$ value of $16 \mathrm{~S}$ rRNA, which reflected the total bacterial count, decreased up to two months; increased at four months; and decreased again at six and 12 months. Therefore, the total bacterial count showed a pattern of increasing first, and then decreasing, and the differences between time point were significant. The $\mathrm{Ct}$ values of $P$. gingivalis, $F$. nucleatum, $P$. intermedia, and T. denticola also showed a pattern of decreasing at two weeks and two months, as compared to the baseline;

Table 1. Change of Cycle Threshold Values in Real-Time Polymerase Chain Reaction on Each Bacteria in the Saliva of 11 Stop-Smokers

\begin{tabular}{lccccccc}
\hline \multicolumn{1}{c}{ Bacteria } & Baseline & 2 weeks & 2 months & 4 months & 6 months & 12 months & p-value \\
\hline 16S rRNA & $19.26 \pm 2.69$ & $18.24 \pm 3.26$ & $17.69 \pm 2.03$ & $21.22 \pm 2.52$ & $16.00 \pm 2.86$ & $17.91 \pm 3.17$ & 0.001 \\
& $(14.3 \sim 21.6)$ & $(11.7 \sim 22.6)$ & $(14.1 \sim 20.6)$ & $(16.9 \sim 25.4)$ & $(12.9 \sim 22.4)$ & $(14.5 \sim 24.5)$ \\
Porphyromonas gingivalis & $35.56 \pm 5.43$ & $30.91 \pm 5.67$ & $32.25 \pm 6.56$ & $36.28 \pm 4.96$ & $31.88 \pm 7.01$ & $31.78 \pm 6.01$ & 0.031 \\
& $(25.3 \sim 40.0)$ & $(23.0 \sim 40.0)$ & $(21.3 \sim 40.0)$ & $(28.5 \sim 40.0)$ & $(21.8 \sim 40.0)$ & $(24.4 \sim 40.0)$ & \\
Fusobacterium nucleatum & $27.05 \pm 2.53$ & $24.48 \pm 3.73$ & $24.91 \pm 3.24$ & $28.32 \pm 4.74$ & $23.18 \pm 2.75$ & $22.77 \pm 1.25$ & 0.001 \\
& $(21.8 \sim 30.7)$ & $(19.3 \sim 31.9)$ & $(18.5 \sim 30.6)$ & $(22.2 \sim 40.0)$ & $(18.4 \sim 26.6)$ & $(21.3 \sim 25.3)$ & \\
Prevotella intermedia & $34.91 \pm 4.97$ & $33.36 \pm 7.38$ & $33.28 \pm 5.79$ & $34.20 \pm 5.12$ & $29.87 \pm 7.39$ & $30.83 \pm 7.05$ & 0.002 \\
& $(28.8 \sim 40.0)$ & $(21.5 \sim 40.0)$ & $(25.80 \sim 40.0)$ & $(27.7 \sim 40.0)$ & $(20.1 \sim 40.0)$ & $(20.9 \sim 40.0)$ & \\
Streptococcus mutans & $33.51 \pm 6.90$ & $33.87 \pm 7.22$ & $30.99 \pm 8.04$ & $36.05 \pm 6.73$ & $34.29 \pm 6.88$ & $35.21 \pm 5.68$ & 0.148 \\
& $(20.8 \sim 40.0)$ & $(23.1 \sim 40.0)$ & $(19.3 \sim 40.0)$ & $(21.8 \sim 40.0)$ & $(22.6 \sim 44.0)$ & $(27.2 \sim 40.0)$ & \\
Treponema denticola & $31.00 \pm 4.86$ & $29.27 \pm 6.10$ & $30.70 \pm 6.30$ & $31.67 \pm 4.80$ & $27.24 \pm 5.27$ & $27.15 \pm 4.78$ & $<0.001$ \\
& $(25.8 \sim 40.0)$ & $(20.9 \sim 40.0)$ & $(23.7 \sim 40.0)$ & $(25.1 \sim 40.0)$ & $(20.4 \sim 40.0)$ & $(23.4 \sim 40.0)$ & \\
Streptococcus sobrinus & $39.04 \pm 3.20$ & $38.61 \pm 4.61$ & $37.64 \pm 7.84$ & $37.93 \pm 4.61$ & $38.66 \pm 4.43$ & $38.98 \pm 3.38$ & 0.493 \\
& $(29.4 \sim 40.0)$ & $(24.7 \sim 40.0)$ & $(14.0 \sim 40.0)$ & $(28.5 \sim 40.0)$ & $(25.3 \sim 40.0)$ & $(28.8 \sim 40.0)$ & \\
\hline
\end{tabular}

Values are presented as mean \pm standard deviation (minimum $\sim$ maximum).

Table 2. Change of Cycle Threshold Values in Real-Time Polymerase Chain Reaction on Each Bacteria in the Gingival Crevicular Fluid of 11 Stop-Smokers

\begin{tabular}{|c|c|c|c|c|c|c|c|}
\hline Bacteria & Baseline & 2 weeks & 2 months & 4 months & 6 months & 12 months & $\mathrm{p}$-value \\
\hline 16S rRNA & $\begin{array}{c}24.72 \pm 2.66 \\
(19.8 \sim 28.8)\end{array}$ & $\begin{array}{c}25.36 \pm 2.12 \\
(21.9 \sim 30.2)\end{array}$ & $\begin{array}{c}25.19 \pm 2.15 \\
(22.8 \sim 29.8)\end{array}$ & $\begin{array}{c}27.14 \pm 3.07 \\
(22.6 \sim 31.3)\end{array}$ & $\begin{array}{c}28.35 \pm 1.76 \\
(24.4 \sim 31.1)\end{array}$ & $\begin{array}{c}28.09 \pm 2.18 \\
(24.7 \sim 30.8)\end{array}$ & 0.001 \\
\hline Porphyromonas gingivalis & $\begin{array}{c}38.11 \pm 3.29 \\
(32.3 \sim 40.0)\end{array}$ & $\begin{array}{l}40.00 \pm 0.00 \\
\quad(40.0)\end{array}$ & $\begin{array}{c}39.14 \pm 2.86 \\
(30.5 \sim 40.0)\end{array}$ & $\begin{array}{c}38.26 \pm 3.01 \\
(32.9 \sim 40.0)\end{array}$ & $\begin{array}{c}39.45 \pm 1.84 \\
(33.9 \sim 40.0)\end{array}$ & $\begin{array}{c}38.47 \pm 2.63 \\
(33.8 \sim 40.0)\end{array}$ & 0.358 \\
\hline Fusobacterium nucleatum & $\begin{array}{c}26.89 \pm 7.16 \\
(21.0 \sim 40.0)\end{array}$ & $\begin{array}{c}26.35 \pm 5.47 \\
(19.6 \sim 40.0)\end{array}$ & $\begin{array}{c}25.93 \pm 5.18 \\
(21.8 \sim 40.0)\end{array}$ & $\begin{array}{c}32.72 \pm 7.22 \\
(22.5 \sim 40.0)\end{array}$ & $\begin{array}{c}34.64 \pm 6.49 \\
(25.6 \sim 40.0)\end{array}$ & $\begin{array}{c}32.10 \pm 7.02 \\
(22.10 \sim 40.0)\end{array}$ & $<0.001$ \\
\hline Prevotella intermedia & $\begin{array}{c}35.46 \pm 5.44 \\
(26.6 \sim 40.0)\end{array}$ & $\begin{array}{c}36.84 \pm 4.49 \\
(29.0 \sim 40.0)\end{array}$ & $\begin{array}{c}35.33 \pm 4.52 \\
(30.4 \sim 40.0)\end{array}$ & $\begin{array}{c}39.08 \pm 3.05 \\
(29.9 \sim 40.0)\end{array}$ & $\begin{array}{c}38.98 \pm 3.38 \\
(28.8 \sim 40.0)\end{array}$ & $\begin{array}{c}38.55 \pm 3.24 \\
(31.7 \sim 40.0)\end{array}$ & 0.090 \\
\hline Streptococcus mutans & $\begin{array}{c}38.83 \pm 2.62 \\
(33.1 \sim 40.0)\end{array}$ & $\begin{array}{c}39.28 \pm 2.38 \\
(32.1 \sim 40.0)\end{array}$ & $\begin{array}{c}39.14 \pm 2.86 \\
(30.5 \sim 40.0)\end{array}$ & $\begin{array}{c}39.54 \pm 1.54 \\
(34.9 \sim 40.0)\end{array}$ & $\begin{array}{c}39.27 \pm 2.41 \\
(32.0 \sim 40.0)\end{array}$ & $\begin{array}{c}40.00 \pm 0.00 \\
(40.0)\end{array}$ & 0.681 \\
\hline Treponema denticola & $\begin{array}{c}34.20 \pm 4.94 \\
(27.6 \sim 40.0)\end{array}$ & $\begin{array}{c}36.93 \pm 4.40 \\
(29.3 \sim 40.0)\end{array}$ & $\begin{array}{c}34.96 \pm 4.19 \\
(28.7 \sim 40.0)\end{array}$ & $\begin{array}{c}37.43 \pm 4.52 \\
(28.7 \sim 40.0)\end{array}$ & $\begin{array}{c}38.94 \pm 3.53 \\
(28.3 \sim 40.0)\end{array}$ & $\begin{array}{c}37.82 \pm 3.74 \\
(31.7 \sim 40.0)\end{array}$ & 0.077 \\
\hline
\end{tabular}

Values are presented as mean \pm standard deviation (minimum $\sim$ maximum). 
increasing at four months; and then decreasing again at six and 12 months. The results were similar to that of $16 \mathrm{~S}$ rRNA and the differences between time point were significant. Meanwhile, S. mutans and S. sobrinus did not show significant differences with respect to time.

The total bacterial count in GCF was lower than in saliva. As shown in Table 2, the Ct values of 16S rRNA in GCF showed an increasing pattern, as compared to the baseline, indicating a decrease in bacterial count and showing significant differences with respect to time. In $\mathrm{GCF}$, the $\mathrm{Ct}$ values of $F$. nucleatum showed significant differences with respect to time. The $\mathrm{Ct}$ value after four months was higher than the $\mathrm{Ct}$ value after 2 months, indicating that the amount of $F$. nucleatum decreased after four months. Other bacteria did not show significant changes and S. sobrinus was detected in none of the GCF samples.

Table 3 shows the results of correlation analysis of $\mathrm{Ct}$ values for each bacterium by time points (results that were not significant are not shown in the table). The correlation coefficients of bacteria at each time point did not show a consistent tendency, with $16 \mathrm{~S}$ rRNA in saliva samples showing positive correlations with different bacteria at different time points. $F$. nucleatum and $P$. gingivalis in saliva samples showed distinctly positive correlations, except at 12 months. $F$. nucleatum and $T$. denticola in saliva samples showed distinctly or strongly positive correlations at all time points. $T$. denticola and $P$. gingivalis in saliva samples showed distinctly positive correlations up to four months. T. denticola in saliva samples showed significantly positive correlations with $P$. intermedia at all time points, except at six months. Meanwhile, 16S rRNA in GCF samples showed strongly or distinctly positive correlations with $F$. nucleatum. Red complex bacteria ${ }^{13)}$ in GCF samples showed correlations at some time points, but the degree of correlation was lower than that of saliva samples. $F$. nucleatum and $T$. denticola in saliva samples showed the strongest correlations.

\section{Discussion}

Although previous studies demonstrated that there are differences in the composition of oral bacteria between smokers and non-smokers, the results were not consistent, and in particular, Prevotella intermedia showed conflicting results with positive correlation with smoking

Table 3. Significant Spearmann Coefficients among Cycle Threshold Values of Oral Bacteria in Saliva and Gingival Crevicular Fluid

\begin{tabular}{|c|c|c|c|c|c|c|}
\hline Bacteria & Baseline & 2 weeks & 2 months & 4 months & 6 months & 12 months \\
\hline 16S rRNA vs. FB & 0.610 & & 0.809 & & & 0.733 \\
\hline 16S rRNA vs. PG & & & 0.740 & & & \\
\hline 16S rRNA vs. TD & & & 0.651 & & & 0.729 \\
\hline 16S rRNA vs. SM & & & & 0.800 & & \\
\hline 16S rRNA vs. PI & & & & 0.616 & & \\
\hline FB vs. PG & 0.676 & 0.740 & 0.744 & 0.683 & 0.767 & \\
\hline FB vs. TD & 0.679 & 0.909 & 0.697 & 0.683 & 0.610 & 0.681 \\
\hline FB vs. PI & & & & 0.763 & 0.834 & \\
\hline PG vs. TD & 0.772 & 0.681 & 0.709 & 0.687 & & \\
\hline PG vs. PI & & & & & 0.840 & \\
\hline TD vs. PI & 0.824 & 0.834 & 0.634 & 0.919 & & 0.726 \\
\hline 16S rRNA_g vs. FB_g & 0.959 & 0.838 & 0.728 & 0.677 & 0.819 & 0.825 \\
\hline 16S rRNA_g vs. TD_g & 0.889 & & & 0.681 & & 0.755 \\
\hline FB_g vs. PG_g & & & & 0.788 & & \\
\hline FB_g vs. TD_g & 0.879 & & 0.751 & 0.788 & & 0.757 \\
\hline PG_g vs. TD_g & & & & 0.956 & & \\
\hline PG_g vs. PI_g & & & & & & 0.669 \\
\hline TD_g vs. PI_g & & & & 0.788 & & 0.857 \\
\hline
\end{tabular}

FB: Fusobacterium nucleatum, PG: Porphyromonas gingivalis, TD: Treponema denticola. SM: Streptococcus mutans, PI: Prevotella intermedia, _. : in gingival crevicular fluid. 
in some cases and negative correlation in others. In a previous study by van Winkelhoff et al. ${ }^{1)}$, six different bacterial strains were analyzed in the periodontal pockets of untreated smokers, untreated non-smokers, treated smokers, and treated non-smokers. The results showed that prevalence of Prevotella intermedia/nigrescens was high in untreated smokers, while average values of Peptostreptococcus micros and Fusobacterium nucleatum were high. Shiloah et al. ${ }^{14)}$ reported that between smokers and non-smokers with no clinical differences, smokers had 18-fold higher likelihood of pathogen colonization and that expression of pathogenic bacteria was proportional to the amount and duration of smoking, even without periodontitis. According to a study on Korean subjects ${ }^{15)}$, Fusobacterium, Fretibacterium, Streptococcus, Veillonella, and Corynebacterium were abundant in smokers, whereas levels of Prevotella, Campylobacter, and Aggregatibacter were low in smokers.

Although the studies on changes in bacterial flora due to smoking have yielded inconsistent results, research on the causes underlying these changes has continued. Wu et al. ${ }^{16)}$ reported that there are differences in the composition of oral microorganisms between current smokers and former smokers, and that metagenome analysis showed that carbohydrate energy metabolism and xenobiotic metabolism are associated with bacterial changes. Shah et al. ${ }^{6}$ reported that exposure to smoking drastically lowered the essential metabolic functions in symbiotic biofilms, whereas it significantly increased the expression of virulent genes, especially those involved in lipopolysaccharide, flagellum, and capsule synthesis.

This study was also conducted with the expectation that the composition of oral bacteria would change in smokers as they go through the process of smoking cessation. Since there was no intervention in the general lifestyle and oral health behaviors of 11 former smokers, these factors are assumed to have been maintained constantly and smoking cessation acted as the intervening factor in this study. Total bacterial count in saliva tended to increase up to two months after smoking cessation; decreased at four months; and increased again from six months onwards. Therefore, a consistent tendency could not be obtained (Table 1), but total bacterial count in GCF continued to decrease according to the duration of smoking cessation (Table 2). These findings were consistent with the results of another study reporting that non-smokers have lower bacterial count $^{14)}$. However, other studies have reported that non-smokers have higher bacterial count in saliva ${ }^{17)}$. Considering that periodontitis starts from the gum ridge, changes of bacteria in GCF would be expected to have a greater effect than changes of bacteria in saliva, and thus, decrease of bacterial count in GCF due to smoking cessation is believed to have a positive effect on periodontal tissues.

$P$. intermedia and $T$. denticola in saliva showed an increasing pattern at six months after smoking cessation. Kubota et al. ${ }^{18)}$ reported that $P$. intermedia and T. denticola are significantly associated with periodontal pocket depth and that smoking and $P$. intermedia are significantly associated with each other. However, the present study showed contradictory results. Meanwhile, a study by Moon et al. ${ }^{15)}$ also reported that the genus Prevotella was less abundant in smokers. Thus, additional studies may be needed to confirm this finding. $F$. nucleatum in GCF showed decrease starting from four months and the changes were significant. A study by Moon et al. ${ }^{15)}$ also found greater amount of $F$. nucleatum in smokers than non-smokers, while the study by Kumar et al. ${ }^{4)}$ reported that Fusobacterium is present in the biofilm of smokers, thereby showing initial inflammatory response. Delima et al. ${ }^{19)}$ reported that after 12 months of smoking cessation and non-surgical periodontal treatment, subgingival microbiome showed increase in non-pathogenic species and decrease in putative periodontal pathogens. The findings of the present study also supported these results. However, there are conflicting results, where Shakhatreh et al. $^{20)}$ reported that level of $F$. nucleatum in gingival sulcus was lower in smokers.

The present study had a small sample size and could not find consistent tendencies in the compositions of bacteria in saliva and GCF. However, the positive correlations between $F$. nucleatum and 16S rRNA in both saliva and GCF and between $F$. nucleatum and $T$. denticola in both saliva and GCF were maintained. Moreover, the results also showed decrease in F. nucleatum in GCF following smoking cessation. Therefore, smoking cessation can 
reduce total bacterial count in GCF and $F$. nucleatum, and thus, can have a positive effect on periodontal tissues. Since decrease in $F$. nucleatum can lead to decrease in $T$. denticola, which showed a positive correlation, it is suggested that red complex oral bacteria that cause periodontitis could be reduced as well following smoking cessation. However, additional studies may be needed on the changing trends of bacteria in saliva and GCF based on smoking.

\section{Notes}

\section{Conflict of interest}

No potential conflict of interest relevant to this article was reported.

\section{Ethical approval}

This study was approved by the Institutional Review Board of Konyang University Hospital (approval no. KYUH 09-25).

\section{ORCID}

Sunghyun Kim, http://orcid.org/0000-0003-2511-6555

Min-Seock Seo, http://orcid.org/0000-0001-7203-7775

Soo-Jeong Hwang, http://orcid.org/0000-0003-4725-1512

\section{Acknowledgements}

This research was supported by Women Scientists Program through the National Research Foundation of Korea (NRF) funded by the Ministry of Science, ICT \& Future Planning (2009-0069179).

\section{References}

1. van Winkelhoff AJ, Bosch-Tijhof CJ, Winkel EG, van der Reijden WA: Smoking affects the subgingival microflora in periodontitis. J Periodontol 72: 666-671, 2001. https://doi.org/10.1902/jop.2001.72.5.666

2. Preshaw PM, Heasman L, Stacey F, Steen N, McCracken GI, Heasman PA: The effect of quitting smoking on chronic periodontitis. J Clin Periodontol 32: 869-879, 2005. https://doi.org/10.1111/j.1600-051X.2005.00779.X

3. Hwang SJ, Kim YK, Yang SJ, Cho HJ: Influence of smoking on matrix-metalloproteinase-9 in the gingival crevicular fluid. J Dent Hyg Sci 11: 339-344, 2011.

4. Kumar PS, Matthews CR, Joshi V, de Jager M, Aspiras M: Tobacco smoking affects bacterial acquisition and colonization in oral biofilms. Infect Immun 79: 4730-4738, 2011. https://doi.org/10.1128/IAI.05371-11

5. Zeller I, Hutcherson JA, Lamont RJ, et al.: Altered antigenic profiling and infectivity of Porphyromonas gingivalis in smokers and non-smokers with periodontitis. J Periodontol 85: 837-844, 2014. https://doi.org/10.1902/jop.2013.130336

6. Shah SA, Ganesan SM, Varadharaj S, Dabdoub SM, Walters JD, Kumar PS: The making of a miscreant: tobacco smoke and the creation of pathogen-rich biofilms. NPJ Biofilms Microbiomes 3: 26, 2017. https://doi.org/10.1038/s41522-017-0033-2

7. Johnson NW: The role of the dental team in tobacco cessation. Eur J Dent Educ 8 Suppl 4: 18-24, 2004.

8. Delima SL, McBride RK, Preshaw PM, Heasman PA, Kumar PS: Response of subgingival bacteria to smoking cessation. $\mathrm{J}$ Clin Microbiol 48: 2344-2349, 2010. https://doi.org/10.1128/JCM.01821-09

9. Liu KH, Hwang SJ: Effect of smoking cessation for 1 year on periodontal biomarkers in gingival crevicular fluid. $\mathrm{J}$ Periodontal Res 51: 366-375, 2016. https://doi.org/10.1111/jre.12316

10. Hyvärinen K, Laitinen S, Paju S, et al.: Detection and quantification of five major periodontal pathogens by single copy gene-based real-time PCR. Innate Immun 15: 195-204, 2009. https://doi.org/10.1177/1753425908101920

11. Wade WG: The oral microbiome in health and disease. Pharmacol Res 69: 137-143, 2013. https://doi.org/10.1016/j.phrs.2012.11.006

12. Bizzarro S, Loos BG, Laine ML, Crielaard W, Zaura E: Subgingival microbiome in smokers and non-smokers in periodontitis: an exploratory study using traditional targeted techniques and a next-generation sequencing. $\mathrm{J}$ Clin Periodontol 40: 483-492, 2013. https://doi.org/10.1111/jcpe.12087

13. Mysak J, Podzimek S, Sommerova P, et al.: Porphyromonas gingivalis: major periodontopathic pathogen overview. J Immunol Res 2014: 476068, 2014. https://doi.org/10.1155/2014/476068

14. Shiloah J, Patters MR, Waring MB: The prevalence of 
pathogenic periodontal microflora in healthy young adult smokers. J Periodontol 71: 562-567, 2000.

https://doi.org/10.1902/jop.2000.71.4.562

15. Moon JH, Lee JH, Lee JY: Subgingival microbiome in smokers and non-smokers in Korean chronic periodontitis patients. Mol Oral Microbiol 30: 227-241, 2015.

https://doi.org/10.1111/omi.12086

16. Wu J, Peters BA, Dominianni C, et al.: Cigarette smoking and the oral microbiome in a large study of American adults. ISME J 10: 2435-2446, 2016.

https://doi.org/10.1038/ismej.2016.37

17. Lira-Junior $\mathrm{R}$, Åkerman $\mathrm{S}$, Klinge $\mathrm{B}$, Boström $\mathrm{EA}$, Gustafsson A: Salivary microbial profiles in relation to age, periodontal, and systemic diseases. PLoS One 13: e0189374,
2018. https://doi.org/10.1371/journal.pone.0189374

18. Kubota M, Tanno-Nakanishi M, Yamada S, Okuda K, Ishihara K: Effect of smoking on subgingival microflora of patients with periodontitis in Japan. BMC Oral Health 11: 1, 2011. https://doi.org/10.1186/1472-6831-11-1

19. Delima SL, McBride RK, Preshaw PM, Heasman PA, Kumar PS: Response of subgingival bacteria to smoking cessation. $\mathbf{J}$ Clin Microbiol 48: 2344-2349, 2010. https://doi.org/10.1128/JCM.01821-09

20. Shakhatreh MAK, Khabour OF, Alzoubi KH, Masadeh MM, Hussein EI, Bshara GN: Alterations in oral microbial flora induced by waterpipe tobacco smoking. Int J Gen Med 11: 47-54, 2018. https://doi.org/10.2147/IJGM.S150553 